\title{
BIM combined with the 3D laser scanning technology to lead the changes of the design industry in the future
}

\author{
Ying Fan $^{1, a^{*}}$, Yan $\mathrm{Shi}^{2, \mathrm{~b}}$ \\ ${ }^{1}$ Huanghe Science and Technology College, Henan, China \\ ${ }^{2}$ Huanghe Science and Technology College, Henan, China \\ a907368490@qq.com, b824786432@qq.com
}

Keywords: BIM technology; 3D laser scanning technology; Point cloud data; Digitization

Abstract. With the advance of BIM technology and the burgeoning popularity of the process, the future of digital design will be gradually digitized by the $\mathrm{CAD}$ drawing gradually evolved into the BIM detailed model information system. Article introduced 3D laser scanning technology in this emerging technology, focus on research through the three-dimensional BIM information of integration and 3D laser point cloud high magnitude, high degree of reduction combined to achieve real and digital conversion efficiency to lead the construction industry development and change in the future of information technology, digital, big data era of transition.

\section{Research Background}

With the growing popularity of BIM technology and process, the design field of the future will be CAD digital drawing has gradually evolved into the BIM information model refinement system, like a dozen years ago, people from hand drawing has gradually evolved into the $\mathrm{CAD}$ digital drawing again the wisdom of the era, the change is coming.

However, this change is not as imagined quickly, just like the original CAD digital evolution of drawings, from the beginning of the introduction of professional and technical personnel to the evolution of the CAD drawing. Now all designers have a CAD drawing capability of the gradual penetration of BIM, also from the simple configuration technology has gradually evolved into the basic skills all employees, but not as a change of place, the evolution of the BIM system, not only from the work on the way to bring a new experience, even from the root layer mode of thinking, influence will also bring no small.

\section{Advantages of BIM System}

The progress of $\mathrm{BIM}$, the first is from $2 \mathrm{D}$ to $3 \mathrm{D}$ progress, let people to express ontology construction in 3D space from 2D using the traditional three view drawings way to building ontology evolution directly using the three-dimensional space model to represent the real three-dimensional space. This performance is direct, without reducing dimension conversion performance, is to reproduce the real, not after refining and processing, any more pure, more primitive.

The progress of BIM, and not just from 2D to 3D progress, is from a traditional Zhang San view individual to a comprehensive unified progress. The expression of conflicts of different drawing related content description may appear in the past, in the BIM center model mode will not appear again. The core is the center of the BIM model, we can see all the drawings, models, parameters table information is extracted from the center of the model, and all of us to extract various information changes, also will change to the real-time center model, and again from the center of the model will be the latest update to extract all from the view on the drawing center model. This is the center model as the core and the foundation, to work on the project itself through the view of terminal project center model. 

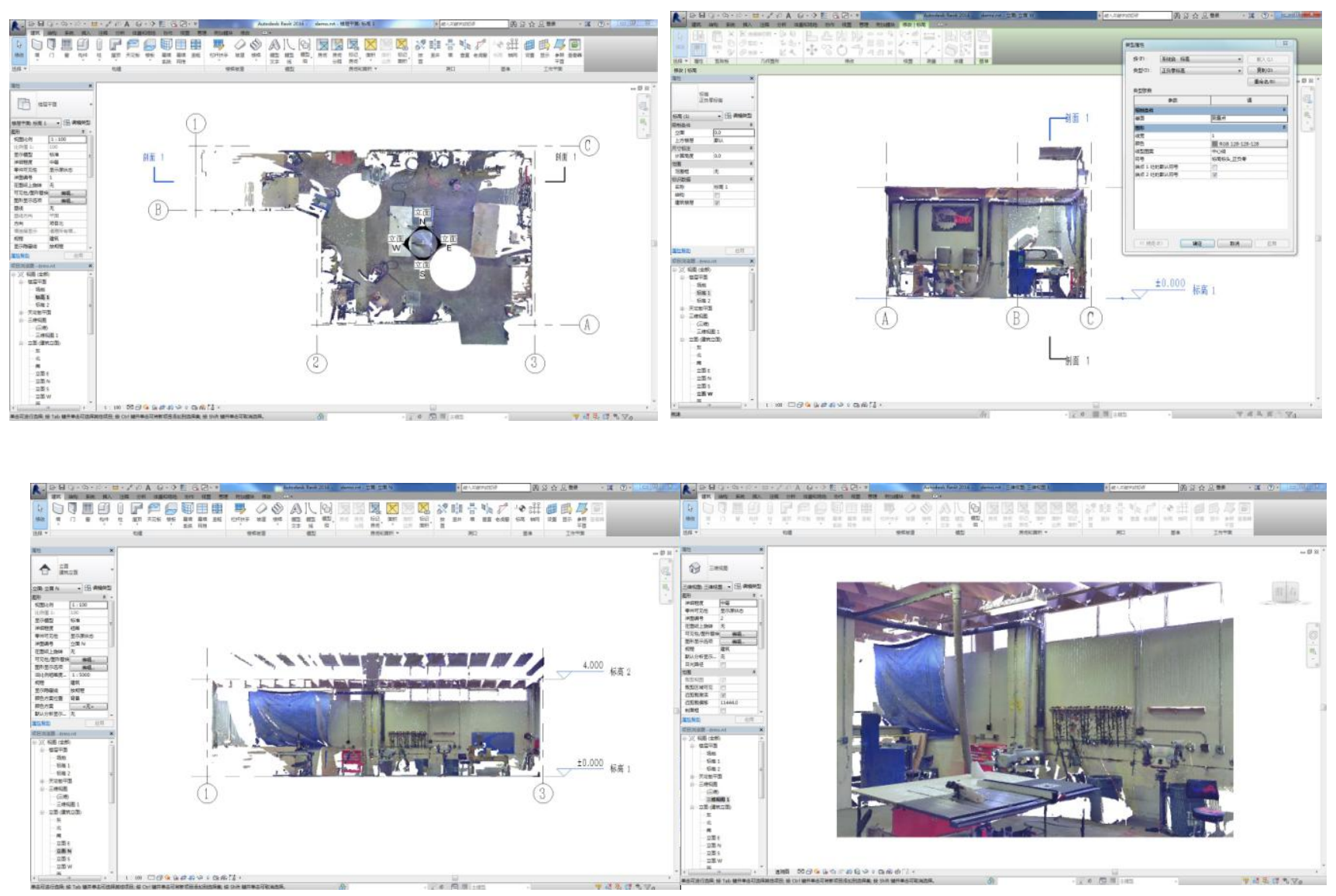

Figure 1. Revit view presentation charts

\section{Laser Scanning Technology}

Definitions

Now the BIM system has been relatively mature, and in many domestic famous cases has rich successful experience, so we do not focus on the superiority of BIM and change the digital, but the introduction of another emerging technology, will lead the industry change -- 3D laser scanning technology.

3D laser scanning technology, also was known as real replication technology. The 3D point cloud data using 3D laser scanning technology,is a collection of hundreds of millions of independent with three-dimensional coordinate information points. A massive point together to form surface, surface combination of adult body, through the set of hundreds of millions of points reflects the space geometry information of the object to be scanned in real three-dimensional space.

3.2 Point Cloud Data Benefits

From the traditional view on the demand of three working mode, 3D point cloud data which have many advantages in the use of. First of all compared to the data obtained by the traditional means of Surveying and mapping, 3D point cloud data is in the amount of information on a higher level, we used by in the process of measurement data from the real key to extracting information can now be at home, because the 3D laser point cloud data back to the real information we can after I get home again from the extraction of real information to the drawing information.

Secondly, from three-dimensional space to two-dimensional orthographic projection of the work can be completed by the computer to. Because the 3D point cloud point is independent, discrete, we can in accordance with their orthographic projection is the way they are rearranged, and projected to a specified projection plane, thus forming in our life can not be observed and photographed the true orthophoto map, 
with color information on RGB cloud data itself with the intensity of the laser reflection information, even through the coaxial lens access, we can even generate visually comparable to the camera shooting out of the picture quality is in a unique way to generate orthophotography true color orthograph.

With this map, we need to actually have the status of traditional surveying and mapping, reconstruction scheme can directly in the color ortho map, like the photos of design work, but because of the point cloud data with real space, so the use of point cloud generated ortho map is also a space size information, reference can be drawn on the amount of orthophoto map directly map. Step back, if there is a situation of drawing demand, we can also paint directly on the status of orthophoto map, which also greatly reduces the status of orthographic drawing difficulty.

\section{Combined with Point Cloud Data and BIM Technology}

\section{Combination Effects}

BIM(Building Information Modeling) is the relevant information and data as a basis for building projects model, building models were set up, true information through digital information simulation building has. It has visualization, coordination, simulation, optimizing and may plot of five characteristics.

American National BIM Standard (NBIMS) definition of BIM, the definition consists of three parts:

1.BIM is a facility (building projects) digital representation of physical and functional properties;

2.BIM is a shared knowledge resource, is a sharing of information about this facility, the facility to provide a reliable basis for the
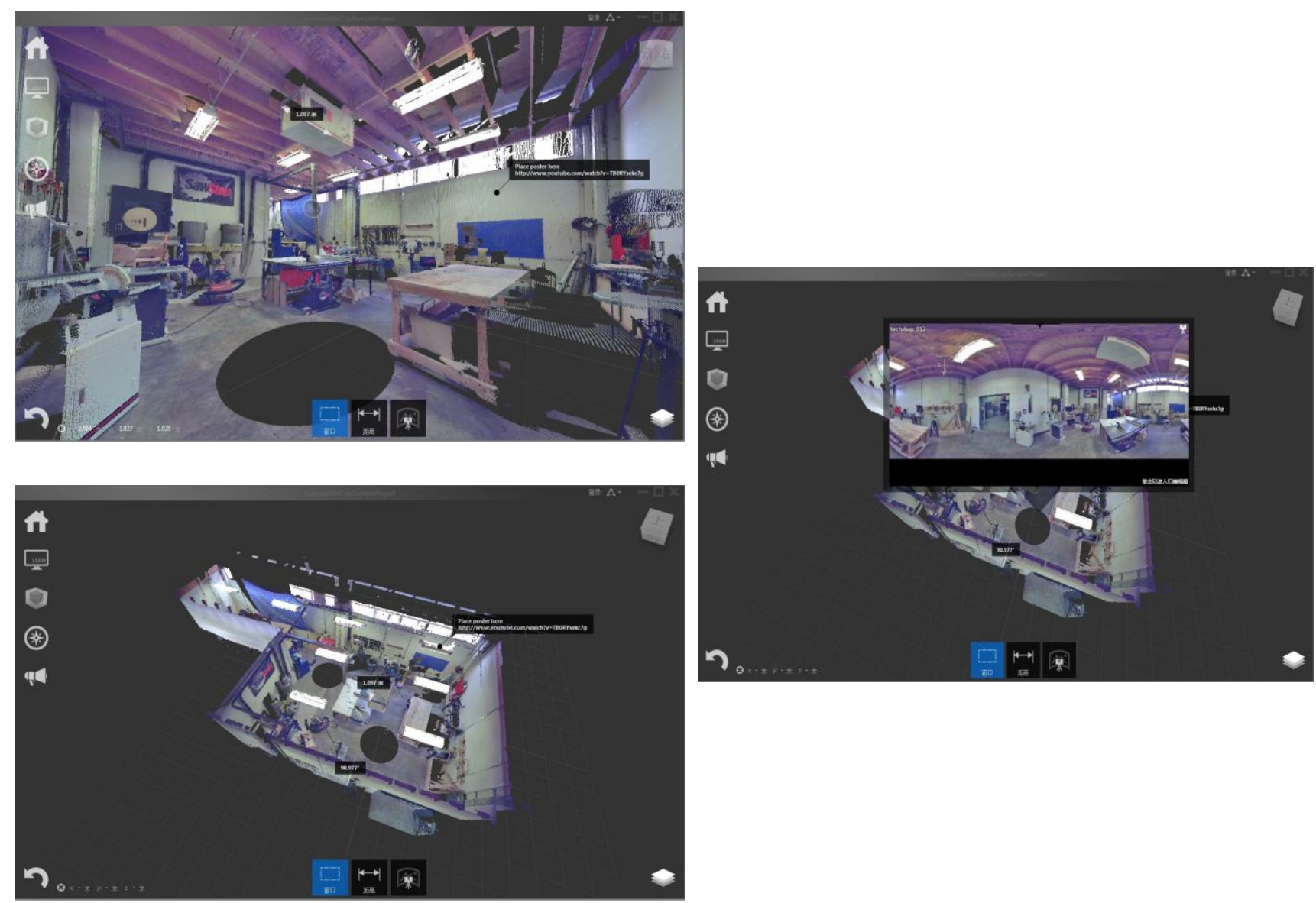

Figure 2. Point cloud presentation charts.

process from construction to demolition of the entire life cycle of all decision-making;

In the different stages of the project, different stakeholders by BIM insert, extract, update and modify information to support and reflect the respective responsibilities of collaboration.

Of course, in the process of extracting spatial information from the design parameters of the real problems encountered, unified data reading, for example, the reality of the wall is not an ideal plane, then the two wall forming corner line is not a standard line. However, in the drawings, if we read this corner line from two 
different views, it is possible or should I say only to extract two different lines, the result is we plot a set of elevation, and that a set of elevation in sizes that are not the same. Unless we when drawing the size all rounded to standard construction module, otherwise the theory we are not possible in the two extraction process to extract the same objective data. This fact itself is from the three-dimensional space of extraction process data to draw relatively independent drawings in the bound to face. We also return to the topic of this paper, combined with the 3D point cloud data and BIM Technology.

Other Aspects

In addition to the design stage, design and construction management of the whole BIM project life cycle properties can also play a role in the construction and management phase using the $3 \mathrm{D}$ point cloud data, such as the construction process on the basis of the construction of point cloud data modification and as in large-scale projects, the installation process using three dimensional laser scanning technique, digital calibration, the installation process virtual assembly, advance the research on the improvement of. The 3D point cloud data as the completion of the final figure also retained, rather than using the completion of drawings more accurately and truly record the true expression of project completion status, which also include the drawing to clearly express the warm wind hydropower pipeline completion status.

\section{Conclusion}

From the point of view, BIM, three-dimensional information integration and high magnitude, 3D laser point cloud with high degree of reduction, is tantamount to combines, from reality to reality from the digital digital, return again, realize the conversion of high degree of binding. In the era of change of the future of digital information, the big data, will lead the development and change of many industries.

\section{References}

[1] Zhang Huixia, Zhu Wenbo . Three-dimensional laser scan data processing theory and application. Electronic Industry Press. 2012.

[2] Xie Hongquan, Hou Kun. Terrestrial laser scanning technology and engineering. Electronic Industry Press. 2012.

[3]Chuck Eastman, Paul Teicholz.BIM Handbook: A Guide to Building Information Modeling for Owners, Managers, Designers, Engineers and Contractors. Wiley.2011.

[4] LIU Guang-wen, MU Pei Chao, Huang Ming-feng. BIM application infrastructure. Tongji University Press. 2013.

[5] Baimu China, the United States Autodesk.Inc companies. Autodesk Revit Architecture 2012 Tutorial official standards. Electronic Industry Press. 2012. 